\title{
UVES radial velocity accuracy from asteroid observations
}

\section{Implications for fine structure constant variability $\star$}

\author{
P. Molaro ${ }^{1}$, S. A. Levshakov ${ }^{2}$, S. Monai ${ }^{1}$, M. Centurión ${ }^{1}$, P. Bonifacio ${ }^{1,3}$, S. D’Odorico ${ }^{4}$, and L. Monaco ${ }^{5}$ \\ 1 INAF - Osservatorio Astronomico di Trieste, via G.B. Tiepolo 11, 34143 Trieste, Italy \\ e-mail:molaro@oats.inaf.it \\ 2 Department of Theoretical Astrophysics, Ioffe Physico-Technical Institute, Polytekhnicheskaya Str. 26, 194021 St. Petersburg, \\ Russian Federation \\ 3 CIFIST, Marie Curie Excellence Team and GEPI, Observatoire de Paris, CNRS, Université Paris Diderot; Place Jules Janssen, \\ 92190 Meudon; Observatoire de Paris, CNRS, 61 avenue de l'Observatoire, 75014 Paris, France \\ ${ }^{4}$ European Southern Observatory, Karl-Schwarzschild-Strasse 2, 85748 Garching bei München, Germany \\ 5 European Southern Observatory, Alonso de Cordova 3107, Casilla 19001, Vitacura, Santiago, Chile
}

Received 17 October 2007 / Accepted 12 December 2007

\begin{abstract}
Context. High resolution observations of the asteroids Iris and Juno have been performed by means of the UV-Visual Echelle Spectrograph (UVES) at the European Southern Observatory (ESO) Very Large Telescope (VLT) to obtain the effective accuracy of the spectrograph's radial velocity. The knowledge of this quantity has important bearings on studies searching for a variability in the fine structure constant carried out with this instrument.

Aims. Asteroids provide a precise radial velocity reference at the level of $1 \mathrm{~m} \mathrm{~s}^{-1}$, which allows for instrumental calibration and the recognition of small instrumental drifts and calibration systematics. In particular, radial velocity drifts due to nonuniform slit illumination and slit optical misalignment in the two UVES spectrograph arms can be investigated.

Methods. We compare the position of the solar spectrum reflected by the asteroids with the solar wavelength positions and with that of asteroid and twilight observations at other epochs to asses the UVES instrumental accuracy.

Results. We observe radial velocities offsets in the range of $\approx 10-50 \mathrm{~m} \mathrm{~s}^{-1}$, likely due to a nonuniform slit illumination. However, no radial velocity patterns with wavelengths are detected and the two UVES arms provide consistent radial velocities. These results suggest that the detected $\Delta \alpha / \alpha$ variability by Levshakov et al. (2007) deduced from a drift of $-180 \pm 85 \mathrm{~m} \mathrm{~s}^{-1}$ at $z_{\text {abs }}=1.84$, between two sets of Fe II lines falling in the two UVES arms, may be real or induced by other kinds of systematics than those investigated here. The proposed technique allows us to make a real time quality check of the spectrograph and should be followed for very accurate measurements.
\end{abstract}

Key words. techniques: radial velocities - instrumentation: spectrographs - standards - minor planets, asteroids galaxies: quasars: absorption lines - Sun: photosphere

\section{Introduction}

Radial velocity precision is required in several fields of astronomical research, ranging from the detection of exoplanets to the study of the variability of the fundamental physical constants. To reveal the presence of an orbiting planet dedicated spectrographs have been manufactured to achieve the best accuracy in the radial velocity. With the High Accuracy Radial velocity Planet Searcher (HARPS) at the $3.6 \mathrm{~m}$ telescope in La Silla, a relative precision of $1 \mathrm{~m} \mathrm{~s}^{-1}$ or higher has been achieved when the full optical stellar spectrum of a solar-type star is recorded and compared in different epochs. A search for a possible variability of the fine structure constant, $\Delta \alpha / \alpha=\left(\alpha_{z}-\alpha\right) / \alpha$, at a redshift $z$, is currently carried out by measuring line shifts between different lines of absorbers observed in spectra of distant quasars that show different sensitivities to $\alpha$ (Webb et al. 1999; Dzuba et al. 2002). Quasars (QSOs) are rather faint and require large telescopes such as the Very Large Telescope (VLT) or the Keck

\footnotetext{
* Based on observations performed at the VLT Kueyen telescope (ESO, Paranal, Chile).
}

combined with the high resolution spectrographs, UVES and the High Resolution Echelle Spectrometer (HIRES) respectively.

By averaging over 143 absorption systems detected in HIRES/Keck telescope spectra of QSOs in a redshift range $0.2<$ $z<4.2$ Murphy et al. (2004) claim that $\Delta \alpha / \alpha=-5.7 \pm 1.1$ parts per million (ppm) implying that in the past the fine structure constant was smaller. On the other hand, no variability has been measured by a different group at the VLT with UVES adopting similar techniques (Quast et al. 2004; Chand et al. 2004; Levshakov et al. 2005, 2006; but see also Murphy et al. 2008; and Srianand et al. 2007). More recently Levshakov et al. (2007) measured a radial velocity difference of $-180 \pm 85 \mathrm{~m} \mathrm{~s}^{-1}$ between Fe II transitions falling in the two different arms of the UVES providing evidence for a variation in the fine structure constant $\Delta \alpha / \alpha=5.4 \pm 2.5 \mathrm{ppm}$, with the fine structure constant being larger in the past at odds with what Murphy et al. (2004) found. Given the importance of these results for fundamental physics a thorough investigation of systematic errors to rule out possible instrumental shifts that may occur during UVES observations is crucial. 
Table 1. Journal of asteroid observations and basic data. Ceres spectra has been observed by HARPS. Expected radial velocities and its components are given in Cols. 6-8. For Ceres, the values refer to midexposure.

\begin{tabular}{lccccccc}
\hline \hline Name & Date & JD & $\begin{array}{c}V \\
\mathrm{mag}\end{array}$ & $\begin{array}{c}\text { Exp. } \\
\mathrm{s}\end{array}$ & $\begin{array}{c}R V_{\text {ast-par }} \\
\mathrm{km} \mathrm{s}^{-1}\end{array}$ & $\begin{array}{c}R V_{\text {ast }-\odot} \\
\mathrm{km} \mathrm{s}^{-1}\end{array}$ & $\begin{array}{c}\Delta R V \\
\mathrm{~km} \mathrm{~s}^{-1}\end{array}$ \\
\hline Iris & $18 / 12 / 06$ & 2454088.518086 & 7.83 & 300 & 12.707 & 1.704 & 14.411 \\
& $22 / 12 / 06$ & 2454092.514539 & 7.95 & 300 & 13.777 & 1.830 & 15.607 \\
& $23 / 12 / 06$ & 2454093.514000 & 7.98 & 300 & 14.030 & 1.862 & 15.892 \\
& $24 / 12 / 06$ & 2454094.515400 & 8.01 & 300 & 14.283 & 1.893 & 16.176 \\
\multirow{4}{*}{ Juno } & $25 / 12 / 06$ & 2454095.513566 & 8.04 & 450 & 14.521 & 1.924 & 16.445 \\
& $24 / 01 / 07$ & 2454125.880451 & 10.62 & 600 & -21.403 & 3.731 & -17.672 \\
& $25 / 01 / 07$ & 2454126.873002 & 10.61 & 900 & -21.364 & 3.721 & -17.643 \\
& $29 / 01 / 07$ & 2454130.873891 & 10.57 & 900 & -21.065 & 3.683 & -17.382 \\
\multirow{4}{*}{ Ceres } & $31 / 01 / 07$ & 2454132.846757 & 10.55 & 900 & -20.949 & 3.664 & -17.285 \\
& $15 / 07 / 06$ & 2453932.837256 & 8.00 & 1800 & -11.288 & 0.456 & -10.832 \\
& $22 / 05 / 06$ & 2453877.919808 & 8.85 & 900 & -22.707 & 0.690 & -22.017 \\
\hline
\end{tabular}

Spectroscopic observations are generally calibrated in wavelength by means of standard calibration lamps, namely the ThAr lamps. However, to achieve a $\Delta \alpha / \alpha$ of $1 \mathrm{ppm}$, a precision of $30 \mathrm{~m} \mathrm{~s}^{-1}$ in the radial velocity of the most sensitive lines is required, challenging the spectrograph precision. Small instrumental effects could be present since the light paths of calibration and stellar beams are different when entering the spectrograph slits. Instrumental flexures, temperatures, and atmospheric pressure instability can produce small radial velocity shifts between calibration and science observations. Temperature and pressure variations as small as $\Delta T=0.3 \mathrm{~K}$ or a $\Delta P=1 \mathrm{mbar}$ produce a drift of $\approx 50 \mathrm{~m} \mathrm{~s}^{-1}$ (Kaufer et al. 2004). These effects can be minimized with the use of ThAr lamps immediately before or after the science exposures if ambient conditions have not changed in the meantime. However, an uneven illumination of the slit may cause spectral shifts and therefore errors in the measurements of radial velocities. This problem is particularly acute in the case of UVES observations with the dichroic mode where the light enters two distinct slits of the two arm spectrograph. Possible effects of different illumination of the two slits of the blue and red arms of UVES are unknown.

To probe small possible instrumental effects in UVES, we observed the solar spectrum reflected by asteroids, which are sources with radial velocities known at the $\mathrm{m} \mathrm{s}^{-1}$ level. This accuracy is not required by the majority of the observations, but is crucial for the investigation of variability of the fine structure constant. The presence of a variability of fundamental dimensionless constants would be a discovery of the outmost importance in theoretical physics with far reaching implications (Copeland et al. 2006; Avelino et al. 2006; Martins 2006; Fujii 2008).

\section{Observations and data analysis}

We collected the observations of two asteroids Iris and Juno and sunlight at twilight, at the VLT with the UVES spectrograph between December 2006 and January 2007, as reported in Table 1. UVES is a two-arm-crossdispersed echelle spectrograph with the possibility to use dichroic beam splitters and to record most of the optical spectrum with one observation (Kaufer et al. 2004). We performed the observations with the dichroic mode, the ESO DIC1, allowing simultaneous observations of the blue and red arms. This dichroic has a cross-over wavelength at $450 \mathrm{~nm}$ and the central wavelengths were set at $390 \mathrm{~nm}$ for the blue and at $580 \mathrm{~nm}$ for the red arms respectively, allowing for full spectral range from $350 \mathrm{~nm}$ up to $680 \mathrm{~nm}$.
We used a 0.5 arcsec slit providing a resolving power of about $\frac{\lambda}{\delta \lambda} \approx 80000$, which is the maximum resolution that can be reached with still adequate sampling of the PSF. It is relevant to note that the target was centered on one of the spectrograph slits, the red slit in our case, while there was no way to check the optical centering on the blue arm slit, directly. The slits were aligned with the parallactic angle to not miss light due to atmospheric diffraction. The two UVES arms are equipped with CCD detectors, one single chip in the blue arm and a mosaic of two chips in the red arm. Each arm has two crossdisperser gratings working in the first spectral order.

Asteroids are apparently fast moving objects and the geocentric radial velocity changes typically by about $1 \mathrm{~m} \mathrm{~s}^{-1}$ in about $4 \mathrm{~min}$, thus also limiting the maximum exposure time with the largest telescopes. At the epoch, Iris and Juno were of 8 and 10.6 mag, respectively, and we kept the exposures at 300 and $900 \mathrm{~s}$ achieving a signal-to-noise between 100-200.

The observations were bracketed by ThAr standard calibration lamps. We carried out calibration and science observations in the attached mode to avoid the automatic resetting of the spectrograph position, implemented by ESO on 26 Dec. 2001, to compensate thermal drifts in the dispersion direction between daytime calibration frames and science observations. The automatic resetting of the instrument allows calibration frames to be taken in daytime economically in terms of observing time, but it makes an accurate calibration problematic.

The data reduction have been performed by means of the UVES Pipeline in the ESO-MIDAS echelle context. The wavelength calibration has been performed using the new atlas of ThAr spectrum by Lovis \& Pepe (2007), which increases the laboratory wavelength precision by means of HARPS observations, with the line selection suggested by Murphy et al. (2007) to avoid blends. Mean residuals of $\leq 0.37 \mathrm{~m} \AA$ for the blue arm; $\leq 0.46 \mathrm{~m} \AA$ for the red low; and $\leq 0.55 \mathrm{~m} \AA$ for the red up; are generally obtained, providing a velocity accuracy at the central wavelengths of $\leq 25 \mathrm{~m} \mathrm{~s}^{-1}$ in the red and of $\leq 30 \mathrm{~m} \mathrm{~s}^{-1}$ in the blue, as shown in detail in Fig. 1. We note that these residuals are about one order of magnitude smaller than those derived by Chand et al. (2006). They can be further improved for limited portions of the spectrum where the reduction is optimized as achieved by Levshakov et al. (2007). As shown by de Cuyper \& Hensberge (1998) an accuracy of $10^{-2}$ of the pixel, which in UVES is $\approx 15 \mathrm{~m} \mathrm{~s}^{-1}$, is attainable for nonblended ThAr lines with more than $10^{3}$ detected electrons in the central pixels. However, the accuracy of the ThAr lines themselves is of the order of 10 to $100 \mathrm{~m} \mathrm{~s}^{-1}$, and this is not directly reflected in the residuals of the wavelength calibration. The reduced spectra have been 


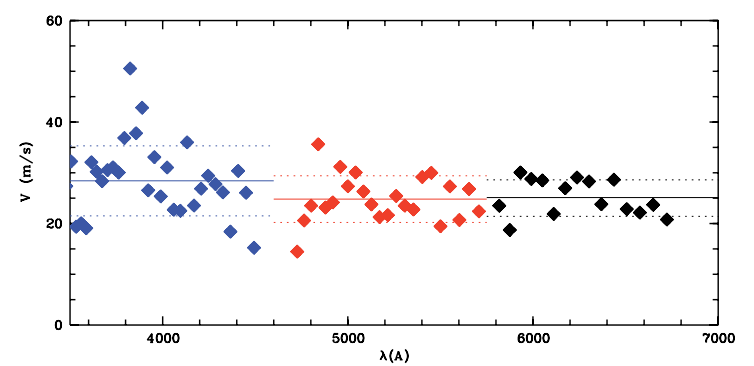

Fig. 1. Typical order residuals of the wavelength calibration, namely the difference between the measured and laboratory wavelength of the ThAr lines used in the calibration. The plotted ones are for Iris 22 Dec. 2006. The three groups, with mean values and $1 \sigma$ dispersion over plotted, refer to the 3 CCDs which have been reduced independently.

normalized manually, tracing the continuum by means of the standard MIDAS routine.

For the reduction of the twilight spectra we skipped the automatic sky subtraction and extracted the spectra from the calibrated frames manually by using the standard MIDAS echelle commands, using a slit height of 8 pixels to minimize effects due to the small curvature of the slit projection on the detector. To check the curvature effects, we extracted 2 spectra from the same image with an extraction slit of 2 pixels, offset by 3 pixels above and below the central position of the order. The position of spectral lines on the two extracted spectra did not reveal notable shifts due to curvature effects.

The angular sizes of the two asteroids in the epoch of observations were of 0.278 arcsec and 0.263 arcsec, and always smaller than the night seeing. They are effective point sources and the light follows the same path through the atmosphere, telescope, and spectrograph not differently from a QSO or other point-like sources. Thus with asteroid observations the radial velocity accuracy could be monitored along the echelle orders for the whole frame in a much better way than with the calibration lamp, since the asteroid lightpath takes into account the atmospheric variations and the centering of the object on the slit. In particular, in the case of UVES observations, which make use of the dichroic, we can monitor the response of the two separate arms. In the twilight spectrum, the diffuse daylight illuminates the slit uniformly so that a comparison between the radial velocity of the asteroid and the daylight probes slit illumination effects on radial velocities.

UVES is also equipped with an iodine absorption cell that can be inserted into the beam to obtain a dense grid of iodine absorption lines superimposed on the target spectrum. The iodine cell currently mounted on UVES produces a rich absorption line spectrum in the range of 490-640 nm. Butler et al. (2004) achieved an accuracy of $0.42 \mathrm{~m} \mathrm{~s}^{-1}$ for UVES with observations of $\alpha$ Cen $\mathrm{A}$, but only after correcting for trends and jumps from a series of 3013 spectra of $1-3$ s exposures. However, the iodine cell is not well suited for measuring accurate positions of QSO absorption lines, which fall very far apart, and we are not aware of its use for this purpose.

\section{Asteroids as radial velocity standards}

Out of 111 stars observed in 20 years with the two Cassegrain spectrophotometers for radial velocities (CORAVEL), only a minor fraction shows a variability of $\approx 200 \mathrm{~m} \mathrm{~s}^{-1}$ (Udry et al. 1999). Thus, radial velocity standard stars provide a reference system of radial velocities with a precision of several hundred $\mathrm{m} \mathrm{s}^{-1}$.
Table 2. Sky observations and data, *spectra taken with HARPS.

\begin{tabular}{lcc}
\hline \hline Date & JD & $\begin{array}{c}R V_{\text {par- } \odot} \\
\mathrm{km} \mathrm{s}^{-1}\end{array}$ \\
\hline $18 / 12 / 06$ & 2454088.480700 & 0.238 \\
$22 / 12 / 06$ & 2454092.481400 & 0.263 \\
$23 / 12 / 06$ & 2454093.479390 & 0.272 \\
$24 / 12 / 06$ & 2454094.478500 & 0.279 \\
$25 / 12 / 06$ & 2454095.480300 & 0.286 \\
$25 / 01 / 07$ & 2454126.472293 & 0.573 \\
$31 / 01 / 07$ & 2454132.482003 & 0.629 \\
$14 / 07 / 06^{*}$ & 2453931.425152 & 0.302 \\
$22 / 10 / 05^{*}$ & 2453666.426383 & -0.070 \\
\hline
\end{tabular}

Among the celestial sources, the asteroids are probably the best radial velocity standard sources for at least two reasons. First they reflect sunlight without any modification of the solar spectrum, and, second, their velocity component with respect to the observer can be predicted with very high accuracy reaching the $\mathrm{m} \mathrm{s}^{-1}$ level (Zwitter et al. 2007).

The first condition is strictly valid only for relatively large asteroids with a nearly spherical shape that produce a constant reflectance of the sunlight. The two selected asteroids Iris and Juno have radii of 99.9 and $117.0 \mathrm{~km}$ respectively and a spherical shape. On the 18 Dec. observation of Iris the illuminated fraction were of $97.07 \%$ and on the 24 Jan. 2007 Juno had a $97.26 \%$ reflectance so that the reflected and the direct solar spectra are likely identical. Variation of reflectance with wavelength or presence of regolith developed by meteoroid impact on the asteroid do not affect high resolution spectra. Also, the asteroid rotation does not affect the solar spectrum and is much smaller than the solar one. The rotational periods for Iris and Juno are 7.14 and $7.21 \mathrm{~h}$ respectively. Thus their rotational velocities would both be of about $25 \mathrm{~m} \mathrm{~s}^{-1}$, which is much lower than the solar rotational velocity and will not cause further significant broadening.

The second reason is that the component of their motion relative to the observer on the earth can be calculated with extreme accuracy. For asteroids with radar monitoring, the orbital computations can take into account the interferences of other bodies of the solar system, including the major asteroids, and reach precisions at the level of the $\mathrm{m} \mathrm{s}^{-1}$ (Zwitter et al. 2007).

Table 1 reports the motion components and the resulting expected radial velocity shifts $\Delta R V$. Ephemeris for our objects has been computed by means of the Jet Propulsion Laboratory's Horizons system $^{1}$, which provides accurate ephemeris for the minor bodies of the solar system. The sunlight reflected by the asteroid is shifted by the heliocentric radial velocity of the asteroid with respect to the sun at the time $t_{1}$ when the photons left the asteroid and further shifted by the component of the earth rotation towards the asteroid at the time $t_{2}$, when the photons reach the earth. The latter shift is the projection along the line-of-sight of the asteroid motion with respect to the observer at the Paranal site adjusted for aberration, and comprises both the radial velocity of the asteroid and the component due to the earth rotation on the line of sight. At Paranal the observed asteroid radial velocity is

$\Delta R V=\left(R V_{\text {ast-par }}+R V_{\text {ast- } \odot}\right)$.

We also take as reference several twilight spectra, which are listed in Table 2. The radial velocity of the skylight reflected by the terrestrial atmosphere is also shifted by the heliocentric

\footnotetext{
1 Available at http://ssd.jpl . nasa.gov/horizons.cgi
} 


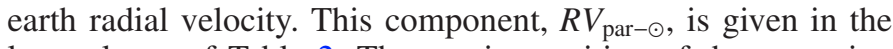
last column of Table 2. The precise position of the scattering of solar light by the atmosphere is not known, but it should be within $10 \mathrm{~km}$ from the ground. In the next sections, we will show that the scattered light from the atmosphere probably shows the transversal motions of the atmosphere, and therefore it is not possible to predict its velocity with the desired accuracy.

\section{Asteroids with UVES}

\subsection{Solar absolute reference}

The highest quality solar spectra in the optical domain are the Fourier Transform Spectrometer (FTS) solar flux and disc-center atlas obtained at the McMath telescope at Kitt Peak by Kurucz et al. (1984) and Brault \& Neckel (1987). These atlases achieve a signal-to-noise ratio of about 2500 with a resolving power of 400000 . Allende Prieto \& Garcia Lopez (1998a,b) used these atlases to measure the central wavelength for a considerable number of lines. Gravitational shifts and convective motions are responsible of line-to-line displacements that can be several hundreds of $\mathrm{m} \mathrm{s}^{-1}$. These displacements vary with the solar cycle showing a modulation with a peak-to-peak variation of $30 \mathrm{~m} \mathrm{~s}^{-1}$ on the 11 years solar activity period with the positions more redshifted in correspondence to the maximum of activity (Deming \& Plymate 1994). However, McMillan et al. (1993) did not reveal any drift within $4 \mathrm{~m} \mathrm{~s}^{-1}$ in the solar line position from a long data series spanning the period from 1987 to 1992. Allende Prieto \& Garcia Lopez (1998a,b) line positions have a precision of the order of $\approx 50-150 \mathrm{~m} \mathrm{~s}^{-1}$ so that they provide absolute reference at this level. The lines formed at the top of the photosphere show shifts close to the gravitational redshift of $636 \mathrm{~m} \mathrm{~s}^{-1}$, while the other lines show the effects of convective motions with variable blue shifts of several hundreds of $\mathrm{m} \mathrm{s}^{-1}$. Lines with equivalent width stronger than $200 \mathrm{~m} \AA$, are rather insensitive to the convective shifts and have been used to estimate the absolute zero of the scale. The value at the plateau level is of $612 \pm 58 \mathrm{~m} \mathrm{~s}^{-1}$ in the case of the solar atlas of Kurucz et al. (1984), which shows the results closest to the theoretical gravitational shift.

We thus compare the measured line positions of the asteroid spectra with the solar line positions provided by Allende Prieto \& Garcia Lopez (1998a) for the solar atlas of Kurucz et al. (1984). In fact the sun light reflected by the asteroids is a sort of integrated solar flux as the Kurucz et al. (1984) atlases. Figure 2 shows the $\Delta R V$ measures for the Iris spectrum of 23 Dec. 2006. The figure shows that there are no major wavelength calibration inaccuracies at the level of $200 \mathrm{~m} \mathrm{~s}^{-1}$ which corresponds to about 0.1 of the pixels size. The result shows a mean value of $\Delta R V=15.614 \pm 0.203 \mathrm{~km} \mathrm{~s}^{-1}$ for the 75 lines measured in the red-up CCD, a $\Delta R V=15.668 \pm 0.234 \mathrm{~km} \mathrm{~s}^{-1}$ for 96 lines in the red-low CCD, and of $15.582 \pm 0.300 \mathrm{~km} \mathrm{~s}^{-1}$ for 63 lines in the blue CCD. Considering that the expected velocity is of $\Delta R V=15.620 \mathrm{~km} \mathrm{~s}^{-1}$, there is an excellent agreement with the red-up CCD and a slight offset of about $50 \mathrm{~m} \mathrm{~s}^{-1}$ and $30 \mathrm{~m} \mathrm{~s}^{-1}$ with the red-low and blue CCD respectively. Despite the scatter of $\approx 200-300 \mathrm{~m} \mathrm{~s}^{-1}$, this analysis shows that there is no significant offset between the two arms of UVES implying that there is no miscentroiding of the target on the two slits of UVES arms.

\subsection{Asteroid versus asteroid}

To overcome the intrinsic uncertainties in the positions of the solar lines, we compared the solar spectrum from an asteroid taken
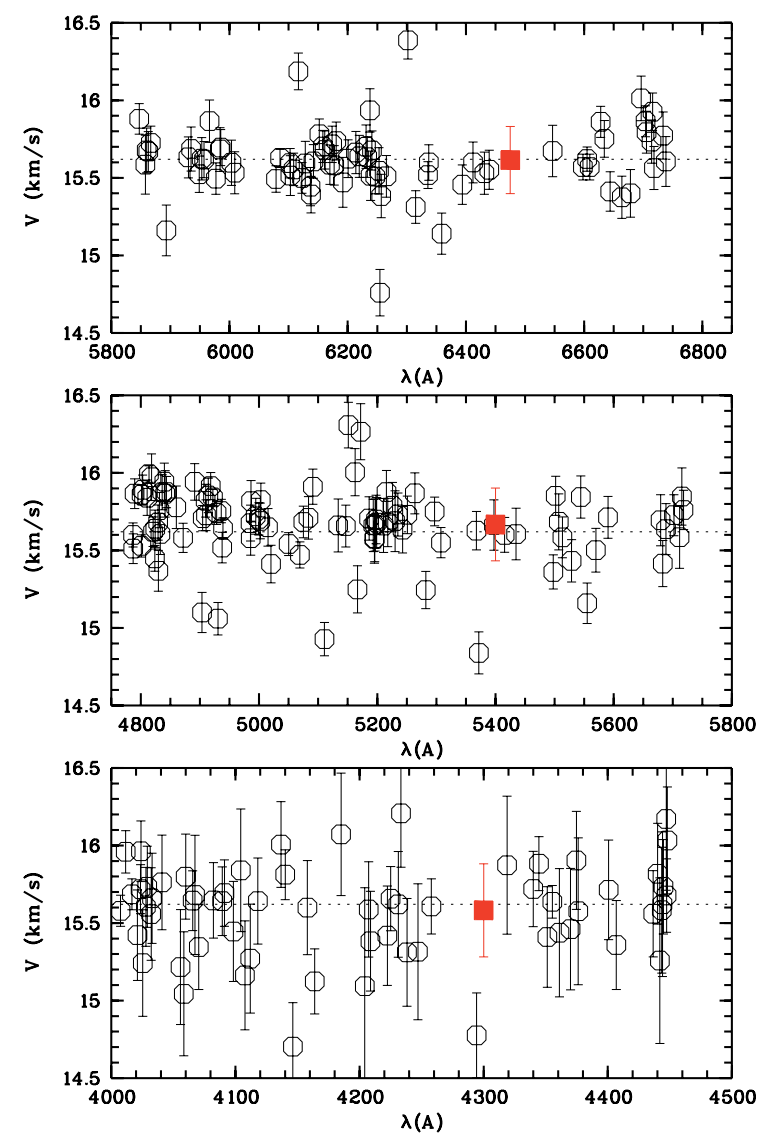

Fig. 2. Line shifts of Iris 23 Dec. 2006 with reference to the Allende Prieto \& Garcia Lopez (1998a) solar line wavelengths, see text for details. The dotted line shows the expected velocity of the asteroid. The top panel refers to the red-up CCD, the middle panel to the red-low $\mathrm{CCD}$, and the bottom panel to the blue CCD. The mean values and their dispersion are shown as squares in the middle of each panel.
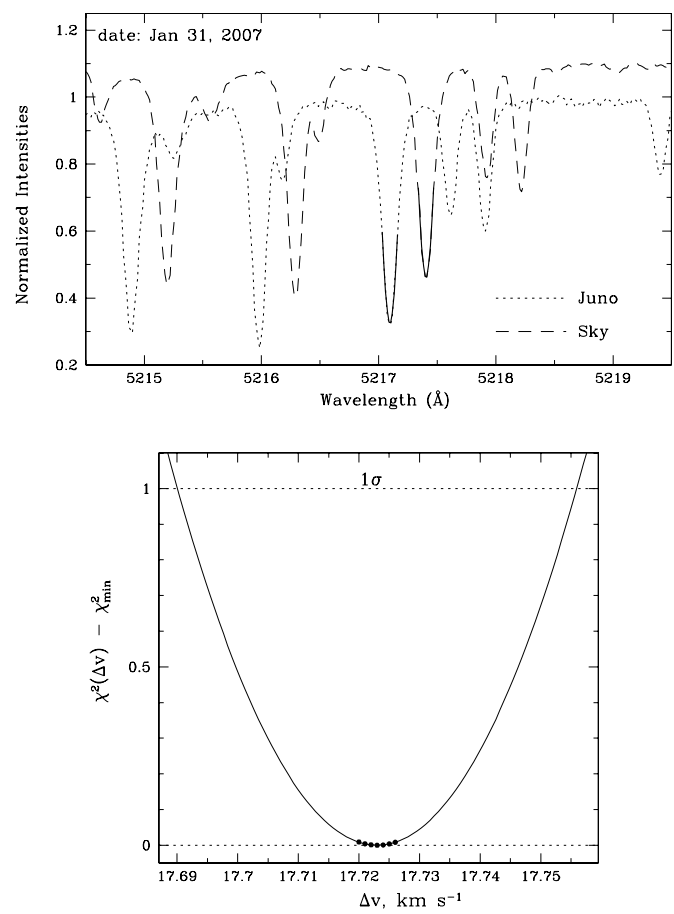

Fig. 3. Procedure for the determination of an accurate $\Delta R V$ and its error. On the top panel, the normalized sky spectrum is slightly shifted vertically for display purposes. 
IRIS $18 / 22$ Dec Blue

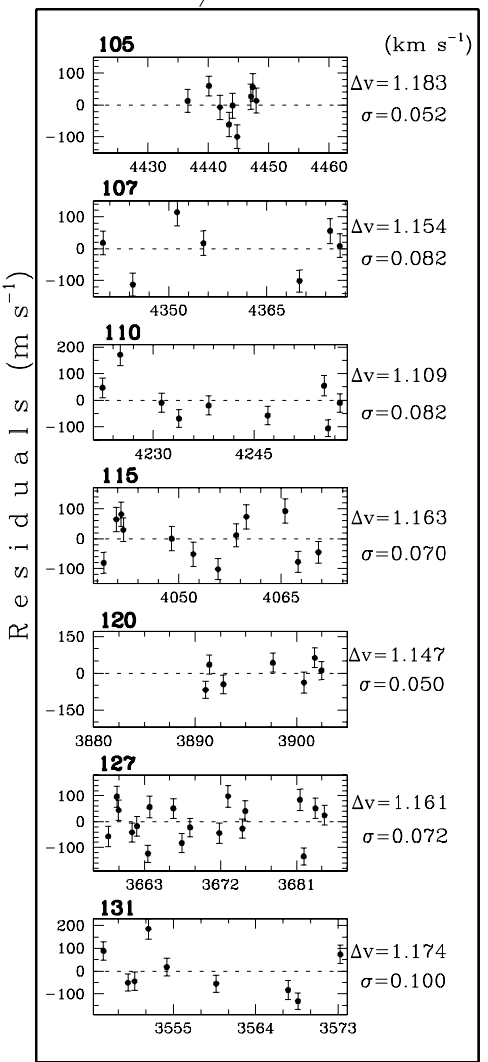

W a v e l e n g t h (A)

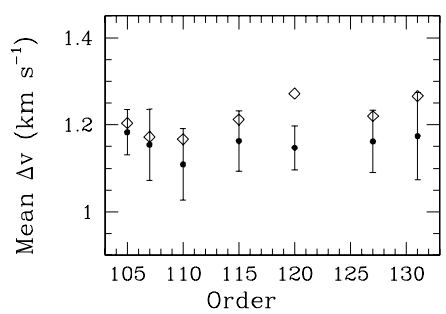

$18 / 22$ Dec Red Low
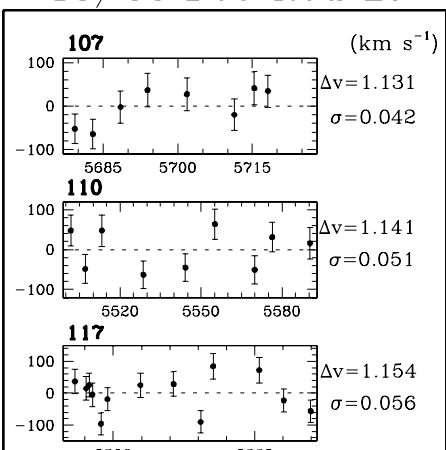$$
122
$$

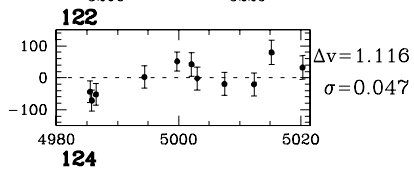

124

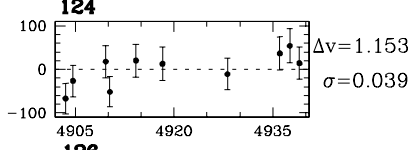

126
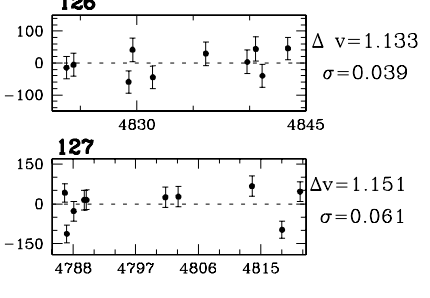

W a v e l e n g t h (A)

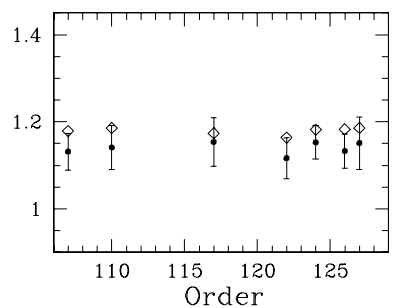

$18 / 22$ Dec Red Up
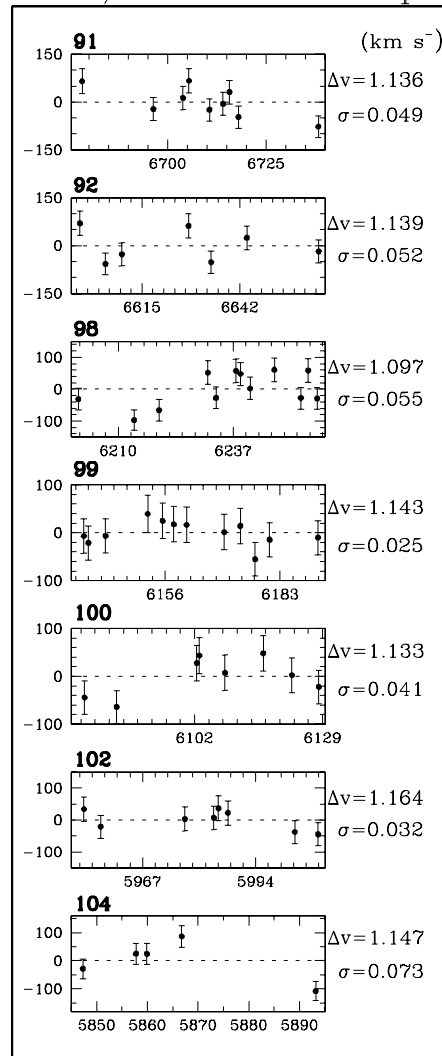

W a v e l e $\mathrm{ng} \mathrm{t} h$ (A)

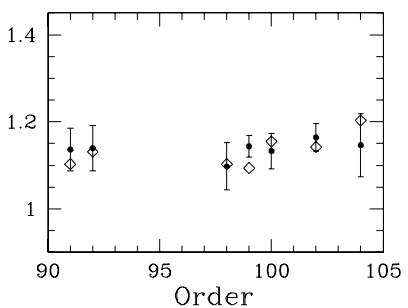

Fig. 4. Radial velocity difference from Iris 18 and 22 Dec. 2006. Residuals correspond to the difference Iris(22) - Iris(18). The predicted $\Delta R V$ is $1.190 \mathrm{~km} \mathrm{~s}^{-1}$. Individual echelle orders (numbered in bold) are shown in the upper panels. For each order, the mean value $\Delta v$ and the sample standard deviation $\sigma$ are indicated. These values $\Delta v$ and $\sigma$ are also shown by dots with error bars in the corresponding low panels where results obtained through the cross-correlation analysis (diamonds) are plotted for comparison.

in two different epochs. In this way we compared each line with itself leaving only the instrumental and calibration imprinting on the change of the asteroid radial velocity between the two epochs.

To accurately measure the radial velocity difference between two lines, we adapted a procedure from Levshakov et al. (2006). We found the most probable $\Delta R V$ between two lines by varying $\Delta R V$ by small incremental steps and estimating the $\chi^{2}$ of the fit. Figure 3 shows a portion of the red-low frame of Juno 31 Jan. and of the sky spectrum of the same day around line 5217.09 $\AA$. The $\mathrm{S} / \mathrm{N}$ are of 126 and 295 for Juno and twilight, respectively calculated from two nearby continuum windows bracketing the line position. The range used in the fitting is marked by thick curves on the upper panel of the figure and we consider only the central parts of the absorption lines to avoid the influence of the wings.

From the fit of points in the vicinity of the global $\chi_{\min }^{2}$ the procedure computes a parabola with the radial velocity difference as variable. We then calculated the $1 \sigma$ uncertainty interval from the parabola when $\chi^{2}(\Delta v)-\chi_{\min }^{2}=1$. For this particular case, we obtained $\Delta R V=-17.723 \pm 0.033 \mathrm{~km} \mathrm{~s}^{-1}$ at $1 \sigma$.

The error is rather typical of our measurements and corresponds to about 0.02 of the pixel size. For instance, in computing the difference between the Iris spectra taken on 18 and 22 Dec. 2006, the 203 lines measured have a mean error of $39 \pm 3 \mathrm{~m} \mathrm{~s}^{-1}$, and of $37 \pm 2 \mathrm{~m} \mathrm{~s}^{-1}$, respectively. The error is mainly photon noise and it depends on the signal to noise ratio of the two spectra. This error sets the precision of our analysis and the level of instrumental effects that can be recovered. In principle, with higher signal-to-noise spectra, this level can be further improved. Wavelength calibration errors are not expected to contribute very much to this error because even if the wavelength calibrations of two spectra are performed independently, they likely make use of the same Ar or Th lines in deriving the calibration coefficients.

The results of the radial velocity difference between the Iris spectra taken on 18 Dec. 2006 with those of 22 and 23 Dec. 2006 are shown in Figs. 4 and 5. The measurements are performed 


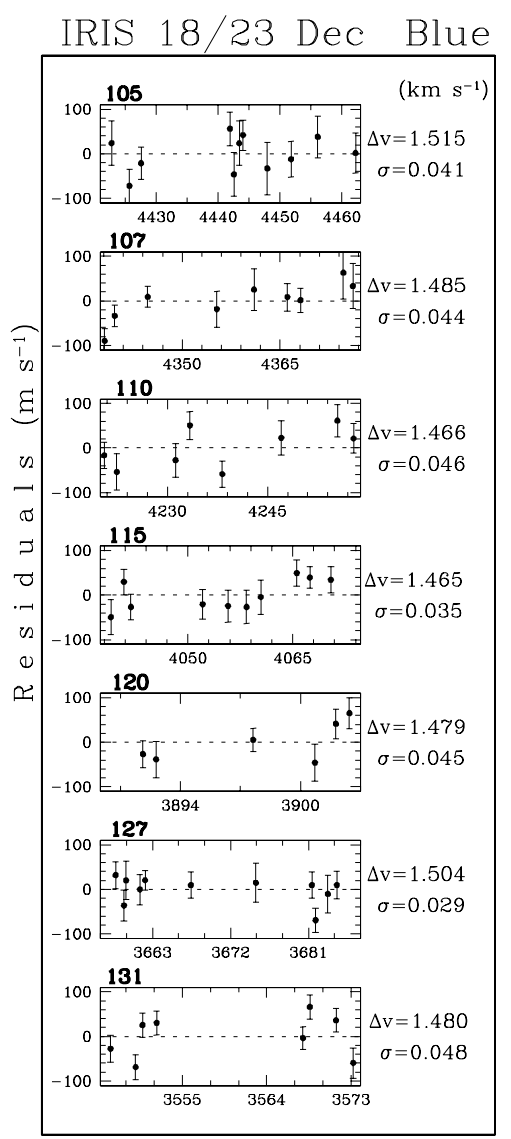

W a v e l e n g t h $(\AA)$

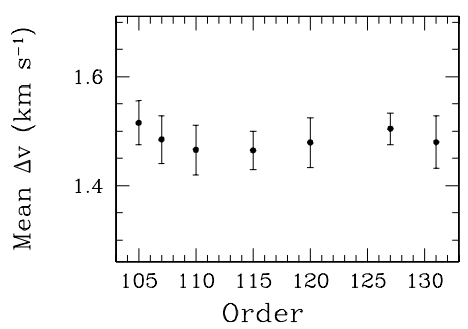

$18 / 23$ Dec Red Low
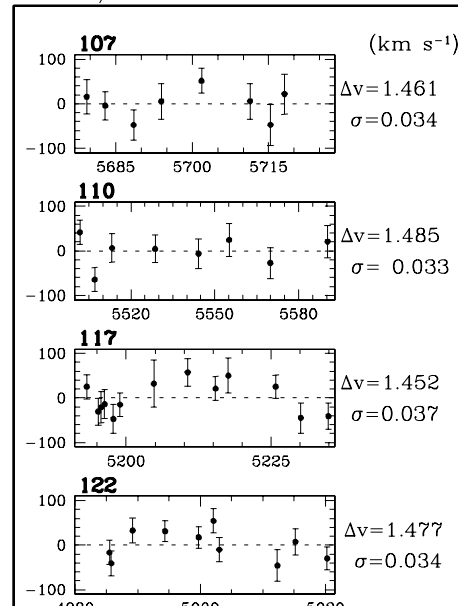

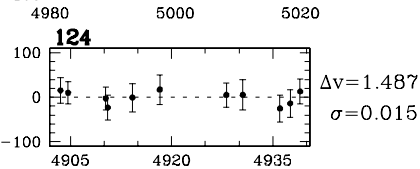

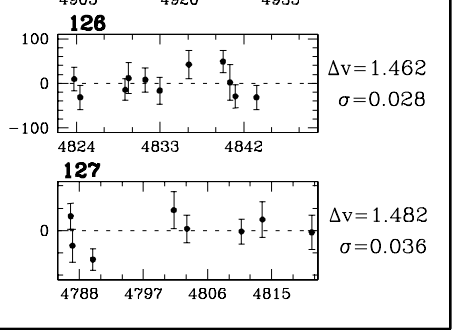

W a v e l e n g t h $(\AA)$
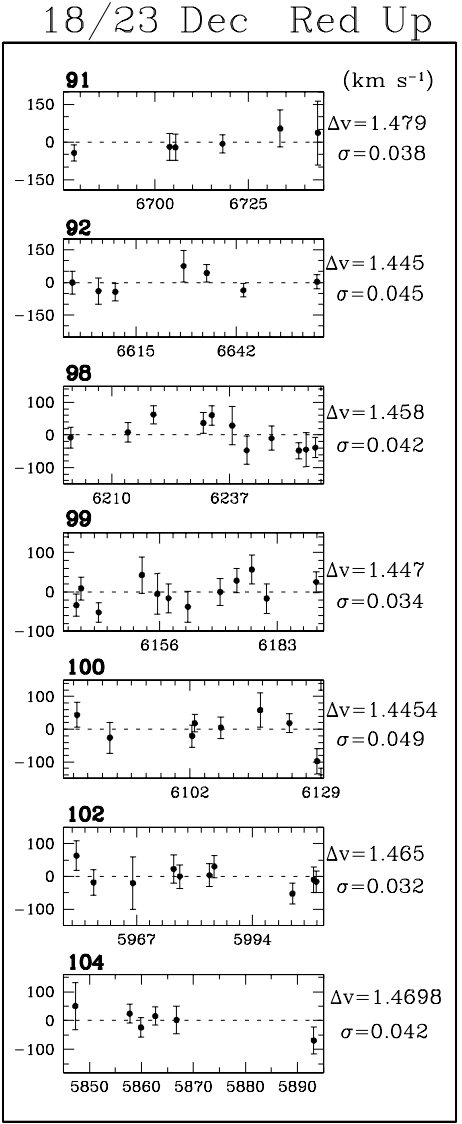

W a v e l e n g t h $(\AA)$
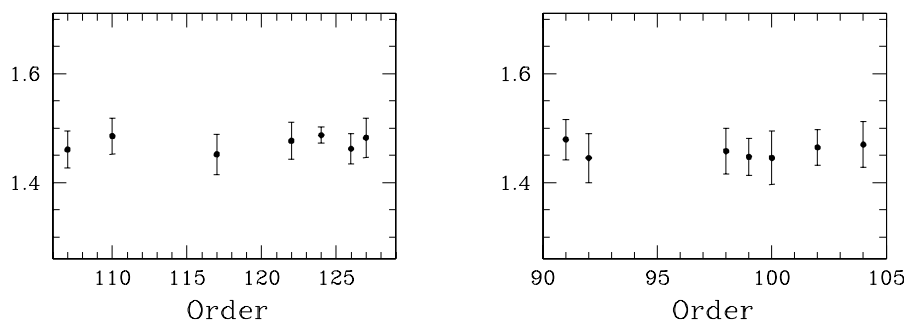

Fig. 5. Same as Fig. 4 but for the comparison between Iris 18 and 23 Dec. 2006. The predicted $\Delta R V$ is $1.447 \mathrm{~km} \mathrm{~s}^{-1}$. Residuals correspond to the difference $\operatorname{Iris}(23)$ - Iris(18).

on lines falling onto 7 orders for each CCD frame selected to map the full spectral range. On the right side of each panel the average value for each single order is reported with the sample standard deviation. As it can be seen from the top panel of the figure, the measurements do not show evidence for trends within an individual order, and the measures are normally distributed around their mean value. At the bottom of the figure the mean values for each order are plotted as a function of the order number. There is no evidence of any pattern of the measured radial velocity with wavelengths from $3500 \AA$ up $6750 \AA$ with measurements involving $3 \mathrm{CCDs}$ and two spectrograph arms. For the 18-22 Dec. 2006 comparison, the mean of the 3 CCDs are $\Delta R V=1.157 \pm 0.073 \mathrm{~km} \mathrm{~s}^{-1}$ for the blue, $1.140 \pm 0.048 \mathrm{~km} \mathrm{~s}^{-1}$ for the red-low, and $1.134 \pm 0.048 \mathrm{~km} \mathrm{~s}^{-1}$ for the red-up. The excess in the dispersion observed within each order reflects the combined contribution of wavelength calibration and data reduction errors with the statistical error. The mean of the mosaic of the two CCDs of the red arm is only $19 \mathrm{~m} \mathrm{~s}^{-1}$ away from the value of the blue arm. For the comparison between 18 and 23 Dec. observations we have a mean value for the blue CCD of $1.487 \pm 0.042 \mathrm{~km} \mathrm{~s}^{-1}$ and for the mean of the two red CCDs a value of $1.464 \pm 0.036 \mathrm{~km} \mathrm{~s}^{-1}$, or $23 \mathrm{~m} \mathrm{~s}^{-1}$ away from the blue arm.

Therefore, there is no evidence for a significant misalignment between the two arms of the UVES spectrograph. However, the expected radial velocity difference is of $1.190 \mathrm{~km} \mathrm{~s}^{-1}$ and of $1.447 \mathrm{~km} \mathrm{~s}^{-1}$, respectively. The mean value of the two arms deviates from the expected velocity by 43 and $24 \mathrm{~m} \mathrm{~s}^{-1}$. This implies a sort of systematic error, which is likely to be ascribed to a nonuniform illumination of the slit in one or in all the observations

Given that there is no evidence for a systematic behavior within the orders in the rest of our observations, we have performed a cross-correlation to get order shifts by means of the IRAF-rvsao XCSAO routine. For this kind of analysis particular care has been adopted in selecting spectral regions without telluric lines that perturb the cross correlation. At the bottom of Figs. 4 and 5 , the measures based on single lines, plotted 
P. Molaro et al.: UVES radial velocity accuracy from asteroid observations. I.

Table 3. Cross-correlation analysis: differential radial velocity shifts measured in $\mathrm{km} \mathrm{s}^{-1}$ with respect to Iris 18 Dec. 2006. The first column reports the echelle orders for the three chips blue, red-low, and red-up. Xcsao $\sigma$ are also indicated.

\begin{tabular}{|c|c|c|c|c|c|c|c|c|c|}
\hline \multirow{2}{*}{$\begin{array}{l}\text { Iris } \\
\text { B }\end{array}$} & \multirow[b]{2}{*}{ range $\AA$} & \multicolumn{2}{|c|}{$18-22 / 12$} & \multicolumn{2}{|c|}{$18-23 / 12$} & \multicolumn{2}{|c|}{$18-24 / 12$} & \multicolumn{2}{|c|}{$\overline{~ 18-25 / 12}$} \\
\hline & & $\mathrm{km} \mathrm{s}^{-1}$ & $\sigma$ & $\mathrm{km} \mathrm{s}^{-1}$ & $\sigma$ & $\mathrm{km} \mathrm{s}^{-1}$ & $\sigma$ & $\mathrm{km} \mathrm{s}^{-1}$ & $\sigma$ \\
\hline 105 & $4420.7-4463.0$ & -1.204 & 0.027 & -1.447 & 0.008 & -1.704 & 0.010 & -2.091 & 0.009 \\
\hline 107 & $4338.5-4379.2$ & -1.172 & 0.046 & -1.450 & 0.008 & -1.706 & 0.010 & -2.085 & 0.009 \\
\hline 110 & $4220.7-4259.2$ & -1.167 & 0.028 & -1.441 & 0.006 & -1.776 & 0.008 & -2.070 & 0.007 \\
\hline 115 & $4038.0-4073.3$ & -1.212 & 0.018 & -1.438 & 0.007 & -1.764 & 0.009 & -2.055 & 0.010 \\
\hline 120 & $3888.0-3902.8$ & -1.272 & 0.030 & -1.476 & 0.012 & -1.761 & 0.012 & -2.051 & 0.011 \\
\hline 127 & $3650.0-3686.9$ & -1.220 & 0.025 & -1.501 & 0.012 & -1.715 & 0.024 & -1.999 & 0.014 \\
\hline 131 & $3540.0-3573.9$ & -1.266 & 0.055 & -1.498 & 0.060 & -1.643 & 0.071 & -2.036 & 0.063 \\
\hline \multicolumn{10}{|l|}{ RL } \\
\hline 107 & $5675.2-5728.5$ & -1.179 & 0.029 & -1.462 & 0.007 & -1.705 & 0.011 & -2.095 & 0.008 \\
\hline 110 & $5521.1-5571.6$ & -1.186 & 0.025 & -1.452 & 0.009 & -1.774 & 0.013 & -2.097 & 0.009 \\
\hline 117 & $5192.2-5236.8$ & -1.174 & 0.021 & -1.403 & 0.007 & -1.763 & 0.011 & -2.071 & 0.012 \\
\hline 122 & $4981.0-5005.0$ & -1.157 & 0.008 & -1.465 & 0.006 & -1.717 & 0.008 & -2.103 & 0.007 \\
\hline 124 & $4900.3-4923.3$ & -1.167 & 0.009 & -1.468 & 0.007 & -1.749 & 0.009 & -2.102 & 0.009 \\
\hline 126 & $4822.8-4845.0$ & -1.183 & 0.041 & -1.461 & 0.008 & -1.726 & 0.011 & -2.084 & 0.009 \\
\hline 127 & $4810.0-4822.8$ & -1.190 & 0.027 & -1.470 & 0.013 & -1.752 & 0.043 & -2.117 & 0.018 \\
\hline \multirow[t]{2}{*}{127} & $4786.4-4805.0$ & -1.183 & 0.024 & -1.436 & 0.012 & -1.740 & 0.020 & -2.135 & 0.026 \\
\hline & & 0.011 & & 0.063 & & 0.039 & & 0.015 & \\
\hline \multicolumn{10}{|l|}{ RU } \\
\hline 91 & $6667.5-6741.2$ & -1.103 & 0.047 & -1.442 & 0.024 & -1.701 & 0.042 & -2.145 & 0.038 \\
\hline 92 & $6595.5-6667.6$ & -1.131 & 0.026 & -1.461 & 0.031 & -1.773 & 0.032 & -2.181 & 0.030 \\
\hline 98 & $6193.7-6257.3$ & -1.103 & 0.012 & -1.463 & 0.011 & -1.777 & 0.014 & -2.120 & 0.012 \\
\hline 99 & $6131.5-6193.7$ & -1.094 & 0.011 & -1.456 & 0.009 & -1.774 & 0.010 & -2.098 & 0.009 \\
\hline 100 & $6077.0-6131.5$ & -1.155 & 0.015 & -1.431 & 0.011 & -1.689 & 0.016 & -2.105 & 0.018 \\
\hline 102 & $5995.0-6010.7$ & -1.142 & 0.017 & -1.452 & 0.022 & -1.741 & 0.027 & -2.144 & 0.027 \\
\hline 104 & $5841.0-5885.0$ & -1.203 & 0.036 & -1.487 & 0.021 & -1.659 & 0.019 & -2.065 & 0.020 \\
\hline \multicolumn{2}{|l|}{ Juno } & \multicolumn{2}{|c|}{$18-24 / 01$} & \multicolumn{2}{|c|}{$18-25 / 01$} & \multicolumn{2}{|c|}{$18-29 / 01$} & \multicolumn{2}{|c|}{$18-31 / 01$} \\
\hline $\mathrm{B}$ & range $\AA$ & $\mathrm{km} \mathrm{s}^{-1}$ & $\sigma$ & $\mathrm{km} \mathrm{s}^{-1}$ & $\sigma$ & $\mathrm{km} \mathrm{s}^{-1}$ & $\sigma$ & $\mathrm{km} \mathrm{s}^{-1}$ & $\sigma$ \\
\hline 105 & $4420.7-4463.0$ & 32.203 & 0.035 & 32.058 & 0.031 & 31.849 & 0.031 & 31.706 & 0.033 \\
\hline 107 & 4338.5 & 32.282 & 0.034 & 32.067 & 0.030 & 31.787 & 0.030 & 31.657 & 0.030 \\
\hline 110 & $4220.7-4259.2$ & 32.249 & 0.051 & 32.044 & 0.049 & 31.839 & 0.051 & 31.648 & 0.050 \\
\hline 115 & $4038.0-4073.3$ & 32.224 & 0.035 & 32.034 & 0.032 & 31.792 & 0.092 & 31.709 & 0.033 \\
\hline 120 & $3888.0-3902.8$ & 32.136 & 0.067 & 32.026 & 0.067 & 31.848 & 0.066 & 31.674 & 0.070 \\
\hline 127 & $3650.0-3686.9$ & 32.183 & & 32.090 & 0.041 & 31.859 & 0.046 & 31.656 & 0.049 \\
\hline 131 & $3540.0-3573.9$ & 32.154 & 0.082 & 32.050 & 0.077 & 31.783 & 0.087 & 31.623 & 0.074 \\
\hline \multicolumn{10}{|l|}{ RL } \\
\hline 107 & $5675.2-5728.5$ & 32.157 & 0.032 & 32.046 & 0.033 & 31.742 & 0.029 & 31.614 & 0.028 \\
\hline 110 & $5521.1-5571.6$ & 32.112 & 0.049 & 32.022 & 0.048 & 31.794 & 0.046 & 31.584 & 0.047 \\
\hline 117 & $5192.2-5236.8$ & 32.086 & 0.037 & 31.995 & 0.034 & 31.789 & 0.038 & 31.577 & 0.037 \\
\hline 122 & $4980.3-5021.3$ & 32.129 & 0.042 & 32.078 & 0.045 & 31.705 & 0.036 & 31.567 & 0.037 \\
\hline 124 & $4900.3-4940.0$ & 32.137 & 0.102 & 31.898 & 0.068 & 31.690 & 0.074 & 31.558 & 0.105 \\
\hline 126 & $4822.8-4845.0$ & 32.123 & 0.106 & 32.013 & 0.101 & 31.739 & 0.103 & 31.648 & 0.105 \\
\hline 127 & $4810.0-4822.8$ & 32.002 & 0.160 & 31.832 & 0.157 & 31.652 & 0.149 & 31.415 & 0.137 \\
\hline 127 & $\begin{array}{l}4785.0-4805.0 \\
31.568\end{array}$ & 32.064 & 0.113 & 31.985 & 0.046 & 31.716 & 0.099 & 31.579 & 0.111 \\
\hline \multicolumn{10}{|l|}{$\overline{R U}$} \\
\hline 91 & $6667.5-6741.2$ & 32.209 & 0.118 & 32.059 & 0.097 & 31.765 & 0.109 & 31.558 & 0.075 \\
\hline 92 & $6595.5-6667.6$ & 32.134 & 0.110 & 31.932 & 0.098 & 31.698 & 0.083 & 31.481 & 0.073 \\
\hline 98 & $6193.7-6257.3$ & 32.109 & 0.040 & 32.012 & 0.041 & 31.809 & 0.036 & 31.658 & 0.035 \\
\hline 99 & $6131.5-6193.7$ & 32.112 & 0.045 & 32.020 & 0.041 & 31.795 & 0.038 & 31.630 & 0.038 \\
\hline 100 & $6077.0-6131.5$ & 32.197 & 0.063 & 32.051 & 0.059 & 31.824 & 0.052 & 31.589 & 0.050 \\
\hline 102 & $5995.0-6010.7$ & 32.184 & 0.049 & 31.978 & 0.042 & 31.741 & 0.048 & 31.610 & 0.037 \\
\hline 104 & $5841.0-5885.0$ & 32.172 & 0.064 & 32.087 & 0.064 & 31.721 & 0.063 & 31.601 & 0.063 \\
\hline
\end{tabular}

in dots, with those performed by means of the XCSAO, plotted in diamonds, show that the two procedures provide consistent results. The results are reported in Table 3 and summarized in Table 4. These measurements performed on the whole set of observations at our disposal confirm that there are not notable patterns with wavelength, no offsets between the two UVES arms and that offsets with respect to the expected velocity in the range $10-50 \mathrm{~m} \mathrm{~s}^{-1}$ are common. 
Table 4. Summary of mean radial velocity shifts measured in $\mathrm{km} \mathrm{s}^{-1}$ with respect to Iris 18 Dec. 2006.

\begin{tabular}{|c|c|c|c|c|c|c|c|c|c|c|}
\hline \multicolumn{11}{|l|}{ Iris } \\
\hline Date & $\Delta R V$ & Blue & $\sigma$ & Red-low & $\sigma$ & Red-up & $\sigma$ & Red & Tot & $\sigma$ \\
\hline $18-22 / 12$ & -1.196 & -1.216 & 0.041 & -1.179 & 0.011 & -1.133 & 0.038 & -1.156 & -1.176 & 0.046 \\
\hline $18-23 / 12$ & -1.481 & -1.464 & 0.027 & -1.474 & 0.063 & -1.456 & 0.018 & -1.465 & -1.463 & 0.039 \\
\hline $18-24 / 12$ & -1.765 & -1.724 & 0.047 & -1.750 & 0.039 & -1.731 & 0.048 & -1.740 & -1.735 & 0.042 \\
\hline $18-25 / 12$ & -2.034 & -2.055 & 0.031 & -2.092 & 0.015 & -2.122 & 0.038 & -2.107 & -2.092 & 0.042 \\
\hline \multicolumn{11}{|l|}{ Juno } \\
\hline Date & $\Delta R V$ & Blue & $\sigma$ & Red-low & $\sigma$ & Red-up & $\sigma$ & Red & Tot & $\sigma$ \\
\hline $18-24 / 01$ & 32.083 & 32.204 & 0.051 & 32.101 & 0.050 & 32.160 & 0.041 & 32.130 & 32.152 & 0.063 \\
\hline $18-25 / 01$ & 32.054 & 32.053 & 0.021 & 31.983 & 0.087 & 32.020 & 0.052 & 32.002 & 32.017 & 0.063 \\
\hline $18-29 / 01$ & 31.793 & 31.822 & 0.033 & 31.730 & 0.052 & 31.765 & 0.047 & 31.747 & 31.770 & 0.058 \\
\hline $18-31 / 01$ & 31.696 & 31.668 & 0.031 & 31.568 & 0.068 & 31.590 & 0.057 & 31.579 & 31.606 & 0.068 \\
\hline
\end{tabular}
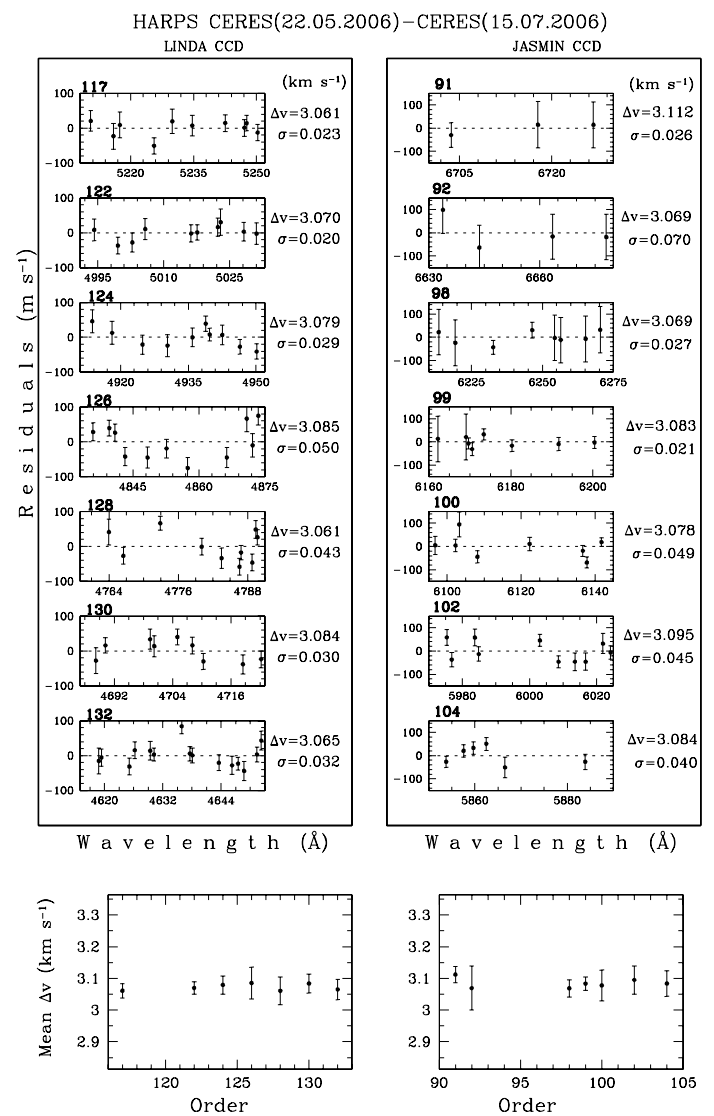

Fig. 6. $\triangle R V$ between Ceres 22 May and 15 July 2006 . The expected $\Delta R V$ is $3.070 \mathrm{~km} \mathrm{~s}^{-1}$.

\section{Asteroids with HARPS}

To check the whole procedure by means of a different instrument specifically designed for high precision radial velocity studies, we retrieved two reduced spectra of Ceres from the public HARPS archive and applied the same kind of measures performed with UVES. The High Accuracy Radial velocity Planet Searcher at the ESO La Silla $3.6 \mathrm{~m}$ telescope is a spectrograph dedicated to the discovery of extrasolar planets through radial velocity oscillations. It is a fibre-fed high resolution echelle spectrograph and is contained in a vacuum vessel to avoid spectral drift due to temperature and air pressure variations. There are two fibers, one collects the star light, while the second is used to record simultaneously a ThAr reference spectrum. Both fibres are equipped with an image scrambler to provide a uniform spectrograph pupil illumination, independent of pointing decentering. In this way the instrument is able to obtain a longterm radial velocity accuracy of the order of $1 \mathrm{~m} \mathrm{~s}^{-1}$ for the entire optical spectrum of a slow rotating G-type star or cooler (Pepe et al. 2005). HARPS has a resolving power of $R \approx 120000$, and provides a sampling of the slit of $F W H M=4.1$ pixels of $15 \mu$ size. Due to the relatively smaller size of the telescope, the exposures are rather long, being namely of $1800 \mathrm{~s}$ and $900 \mathrm{~s}$ (see Table 1). In the course of the exposure the radial velocity of the asteroid changes by $\approx 50$ and $25 \mathrm{~m} \mathrm{~s}^{-1}$, respectively. The expected velocities reported in Table 1 refer to the midexposure times.

In Fig. 6 we give the radial velocities measured between the observations of Ceres taken on 22 May 2006 and on 15 July 2006. The accuracy of the measure of a pair shift is now better than $\approx 20 \mathrm{~m} \mathrm{~s}^{-1}$ and the line-to-line variation of the positions is almost entirely due to errors in the wavelength calibration. The mean of the blue CCD Linda is $\Delta R V=3.072 \pm$ $0.010 \mathrm{~m} \mathrm{~s}^{-1}$, and the mean of the red CCD Jasmin is $3.080 \pm$ $0.010 \mathrm{~m} \mathrm{~s}^{-1}$. The predicted $\Delta R V$ shift is of $3.070 \mathrm{~km} \mathrm{~s}^{-1}$ and is found in excellent agreement with the measured velocity within few $\mathrm{m} \mathrm{s}^{-1}$. This suggests that the systematic offset observed in the UVES spectra is related to the slit acquisition mode which remains the most significant observational and technical difference between the two spectrographs.

\section{Twilight}

Sky observations differ from point source observations mainly because the slit is uniformly illuminated. Thus, a differential measure of a point-like source as an asteroid with the same feature as the sky spectrum in principle allows us to probe radial velocity drifts induced by a nonuniform slit illumination. The results of the measures for the Juno observations of 31 Jan. are shown in Fig. 7. The observations of Juno on 31 Jan. when compared with the skylight on $31 \mathrm{Jan}$. show that the $\Delta R V$ inferred from the three CCDs are all consistent with each other. The blue CCD gives a mean value of $\Delta R V=-17.797 \pm 0.064 \mathrm{~km} \mathrm{~s}^{-1}$; the red-low a $\Delta R V=-17.757 \pm 0.050 \mathrm{~km} \mathrm{~s}^{-1}$; and the redup a $\Delta R V=-17.772 \pm 0.057 \mathrm{~km} \mathrm{~s}^{-1}$. The expected velocity is of $\Delta R V=-17.914 \mathrm{~km} \mathrm{~s}^{-1}$, therefore, we observed an offset of $\approx 150 \mathrm{~m} \mathrm{~s}^{-1}$. This offset is rather high and about a factor three higher than the offset observed in the series of asteroid-asteroid comparison. To check the procedure we performed two separate tests. In one test, we compared the accurate HARPS observations of Ceres with a sky spectrum taken with the same instrument, and in a second test we compared two twilight spectra taken with UVES in two different epochs. 
JUNO 31 Jan Blue

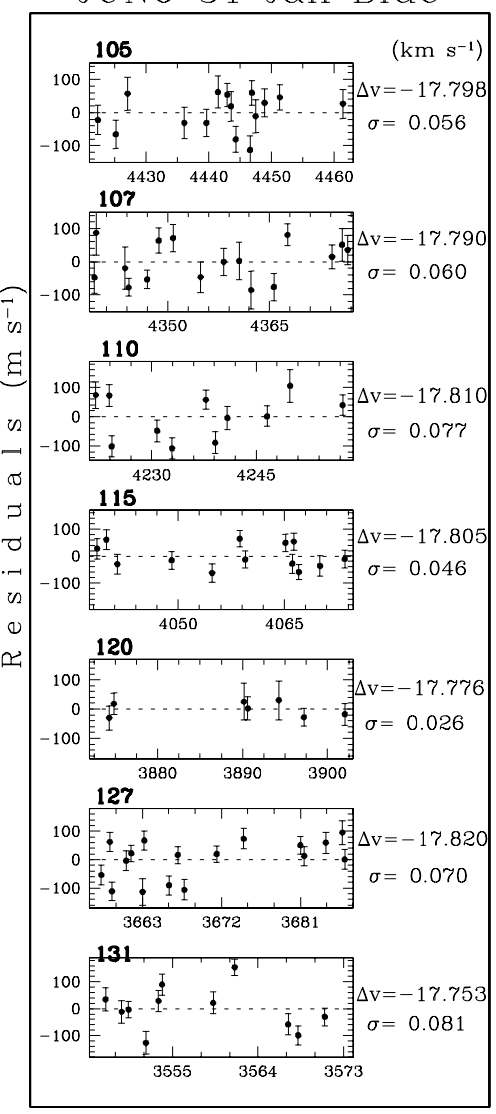

W a v e l e n g t h $(\AA)$
31 Jan Red Low

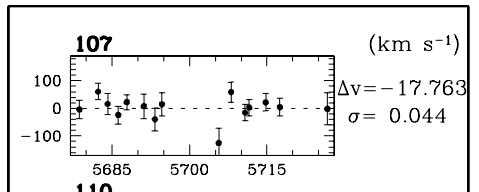

110

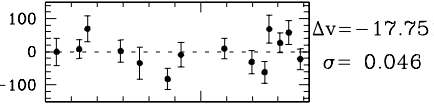

5520
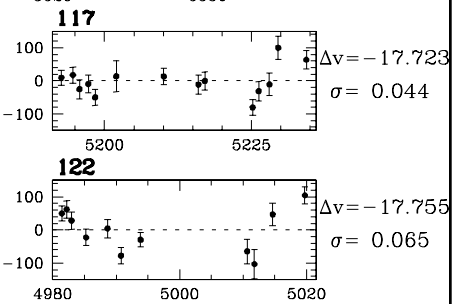

124

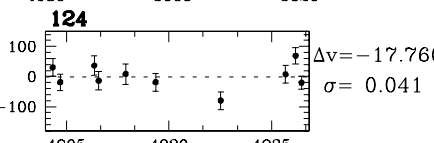

126

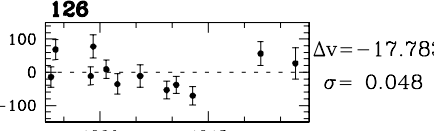

$4830+4845$

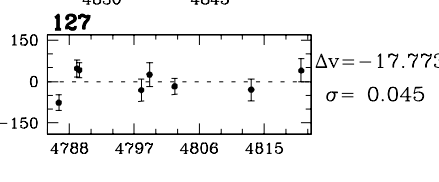

W a v e l e n g t h $(\AA)$

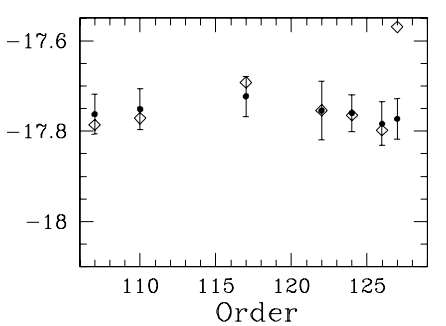

31 Jan Red Up
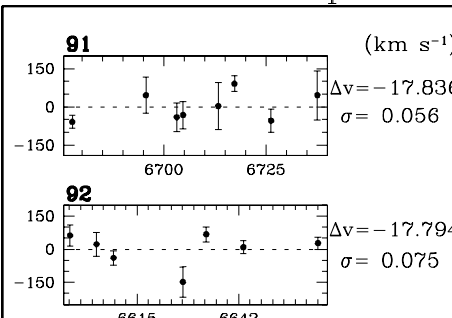

$986615-6042$

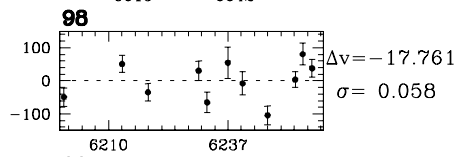

$99^{62}$
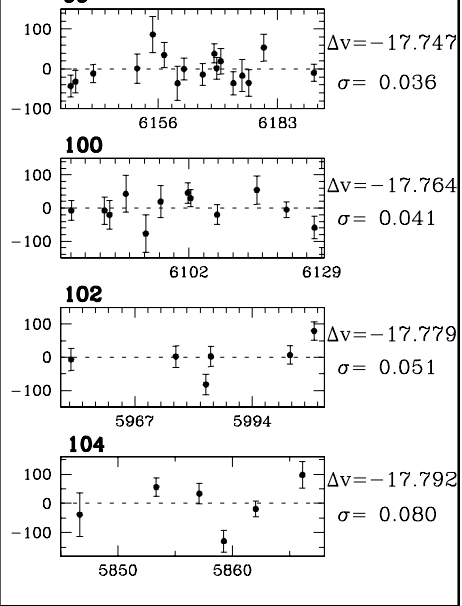

W a v e l e n g t h $(\AA)$

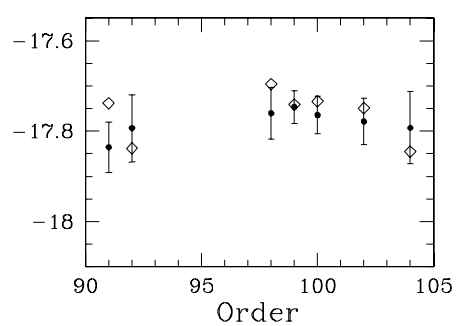

Fig. 7. Juno, 31 January relative to the twilight of the same date. Residuals correspond to the difference Juno - Sky.

In Fig. 8, we show the comparison between the spectrum of Ceres taken on 15 July 2006 with a sky light taken with the same instrument on 22 Oct. 2005. The mean $\Delta R V$ values in the two HARPS CCDs are $\Delta R V=23.657 \mathrm{~km} \mathrm{~s}^{-1}$ for the blue CCD Linda, and $\triangle R V=23.647 \mathrm{~km} \mathrm{~s}^{-1}$ for the red CCD Jasmin, while the predicted one is of $\Delta R V=23.709 \mathrm{~km} \mathrm{~s}^{-1}$ computed for the middle Ceres's exposure. This measure shows an offset of $\approx 50 \mathrm{~m} \mathrm{~s}^{-1}$ between the Ceres and sky spectrum. This offset is not observed when the Ceres of two epochs are compared with each other, as we discussed in the previous section. HARPS is fed by fiber optics and therefore we expected no difference between the two kinds of measures suggesting that the sky spectrum holds a component of motion of several tens of $\mathrm{m} \mathrm{s}^{-1}$. This implies that the twilight solar spectrum is not a good reference for the determination of the zero scale.

As a second test we compared the sky spectra with each other. The difference between the sky spectra taken with HARPS on 22 May 2005 and 14 July 2006 are shown in Fig. 9. The expected velocity difference for this pair is of $-371 \mathrm{~m} \mathrm{~s}^{-1}$ while the mean value is $-275 \pm 39 \mathrm{~m} \mathrm{~s}^{-1}$. Thus, also in this case, we fail to reproduce the expected velocity confirming that the sky spectrum is sensitive to unpredictable motions likely due to currents in the upper terrestrial atmosphere.

We also emphasize that close inspection of UVES twilight and asteroid solar spectra show that they are not completely identical. Small differences at the level of $1-2 \%$ are found between the twilight spectrum and the asteroid reflected solar spectrum consistent with the findings of Zwitter et al. (2007). An example of the two spectra, with the skylight lines shallower, is shown in Fig. 10. Similar differences have been found also by Gray et al. (2000) and also depending on the angular separation from the Sun. According to Gray et al. (2000), the skylight variations can be explained as a combination of Rayleigh-Brillouin scattering with a second term of aerosol. The measurements of the full width at half maximum $(F W H M)$ for a representative sample of lines of asteroid and twilight spectra are shown in Fig. 11. The $F W H M$ of the twilight are slightly larger by about $5 \mathrm{~m} \AA$ with comparison to the asteroid ones. This suggests the presence of turbulence in the atmospheric layers that reflect the sun light. At twilight, a transverse motion in the atmosphere has 


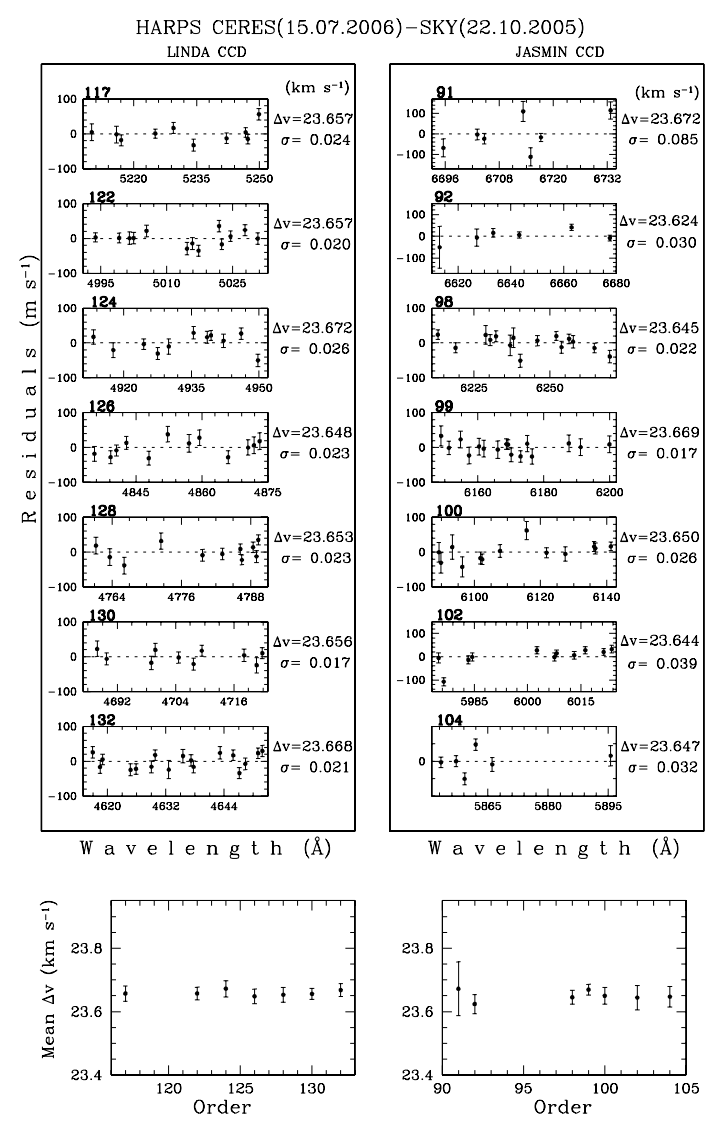

Fig. 8. $\Delta R V$ between Ceres 15 July 2006 and the Sky on 22 Oct. 2005. The predicted $\Delta R V$ is $23.709 \mathrm{~km} \mathrm{~s}^{-1}$.

a considerable component in the direction of the sun and produces a radial velocity drift in the reflected spectrum. A detailed investigation of these effects is beyond the scope of this paper, but the presence of these effects shows that the twilight spectrum is not a good zero reference point at the level of $\approx 100 \mathrm{~m} \mathrm{~s}^{-1}$.

\section{Implications for $\Delta \alpha / \alpha$}

In the Many Multiplet method, the measurability of $\Delta \alpha / \alpha$ from observations of absorption lines in QSO spectra is based on the fact that the energy of each line transition shows a different sensitivity to a change of $\alpha$ (Webb et al. 1999). Thus, the value of $\Delta \alpha / \alpha$ depends on the measure of the relative radial velocity shifts, $\Delta R V$, between lines with different sensitivity coefficients. The relation between the radial velocities and $\Delta \alpha / \alpha$ is (Levshakov et al. 2006):

$\left(v_{2}-v_{1}\right)=2 c\left(Q_{1}-Q_{2}\right) \frac{\Delta \alpha}{\alpha}$,

where $Q$ is the sensitivity coefficient $Q=q / \omega_{0}$, with $\omega_{0}$ being the frequency and $q$ the theoretical so-called $q$-factor. The $q$-factors have been computed for the most important UV resonance transitions by Dzuba et al. (2002) and the $q$-factors for Fe II were re-calculated by Porsev et al. (2007).

The largest $\Delta Q$ is presently provided by the Fe II resonance lines. By comparing the Fe II $\lambda 1608$ with and Fe II $\lambda 2382$ or $\lambda 2600$ lines $\left(Q_{1608}=-0.0166 ; Q_{2382}=0.0369\right.$; and $Q_{2600}=$ 0.0367 from Porsev et al. 2007) we obtain $|\Delta Q| \simeq 0.053$, which is almost two times larger than that obtained from a combination of other transitions. In this case, a shift of $\approx 30 \mathrm{~m} \mathrm{~s}^{-1}$ between the Fe II lines corresponds to a $\Delta \alpha / \alpha$ of $\approx 1 \mathrm{ppm}$.

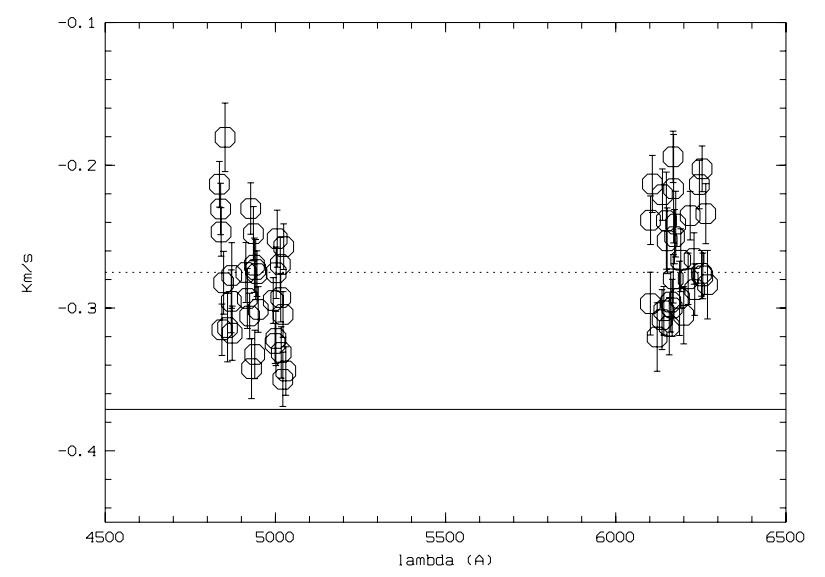

Fig. 9. $\Delta R V$ between HARPS sky of 22 May 2005 and 14 July 2006. The dotted line is the mean value while the red line shows the expected $\Delta R V$ at $-371 \mathrm{~m} \mathrm{~s}^{-1}$.

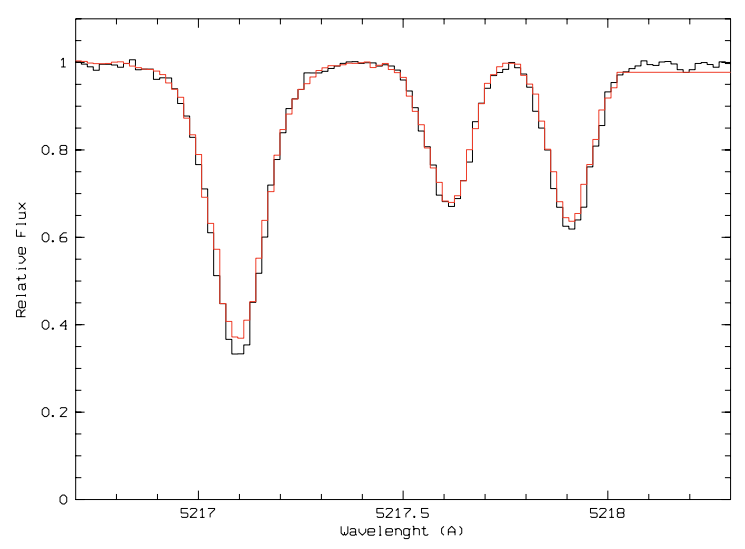

Fig. 10. Portion of the asteroid (thick line histogram) and twilight (thin line histogram) spectrum around the line $5217.3 \AA$.

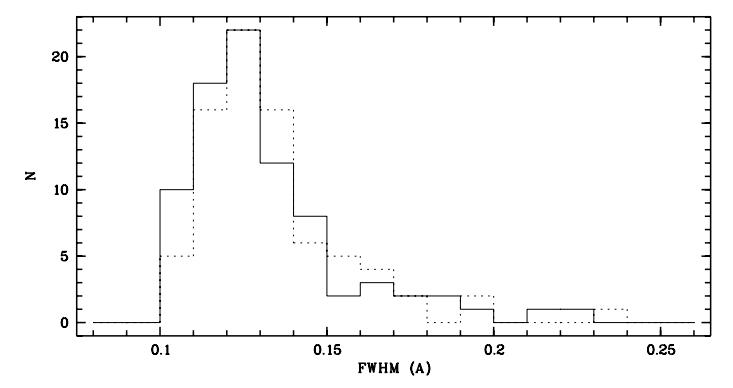

Fig. 11. FWHM for asteroid Juno (solid line) and twilight (dashed line).

Levshakov et al. (2007) analyzed Fe II profiles associated with the $z_{\mathrm{abs}}=1.84$ Damped Ly $\alpha$ system from UVES observations of the quasar Q 1101-264. The data represent one of very few spectra of QSOs obtained with spectral resolution $F W H M$ of $3.8 \mathrm{~km} \mathrm{~s}^{-1}$ and $S / N>100$. In this work, a shift of the relative radial velocity between the $\lambda 1608$ and $\lambda \lambda 2382$, 2600 lines of $\triangle R V=-180 \pm 85 \mathrm{~m} \mathrm{~s}^{-1}$ was obtained. With the updated sensitivity coefficients from Porsev et al. (2007) this shift in the radial velocity between the Fe II lines corresponds to a $\Delta \alpha / \alpha=5.66 \pm 2.67 \mathrm{ppm}$.

The Fe II lines fall at $\lambda \sim 4566 \AA$ and $\lambda \sim 6765,7384 \AA$, respectively, which is quite far apart in the two different UVES arms so that a hidden systematic effect would challenge the interpretation as due to variation of $\alpha$. Levshakov et al. (2007) measured the same velocity between the $\lambda 2382$ and $\lambda 2600$, which is 
what expected since the $Q$ values for $\lambda 2382$ and $\lambda 2600$ are about equal. However, there is no direct way to check out systematic differences of the Fe II $\lambda 1608$ with lines that fall in the other arm of the spectrograph. Different velocity offsets may occur in the blue and red frames causing an artificial Doppler shift between the Fe II $\lambda 1608$ and $\lambda \lambda 2382,2600$ lines and mimicking a change in $\Delta \alpha / \alpha$. The set of measures carried out here show that there are no $\triangle R V$ offsets between the two UVES arms greater than $30 \mathrm{~m} \mathrm{~s}^{-1}$. This excludes this kind of systematics as a possible origin of the signal detected by Levshakov et al. (2007). Therefore, either the detection is real or it is induced by a systematics of different kind.

\section{Conclusions}

Observations of asteroids have been conducted with the UVES spectrograph at the VLT to probe the radial velocity accuracy achievable with the spectrograph. By means of HARPS observations, we have shown that the asteroid observations are excellent radial velocity standards able to probe the instrumental accuracy in any particular position of the spectrum down to the limit provided by the ThAr wavelength calibration, or $10 \mathrm{~m} \mathrm{~s}^{-1}$.

By comparing the asteroid line positions with the absolute ones from solar positions, which account for solar convective shifts, we have shown that the UVES spectrograph is not affected by any systematics along the whole optical domain at the level where the solar line positions are known, namely of few hundreds of $\mathrm{m} \mathrm{s}^{-1}$. We have further refined the analysis by comparing asteroid observations at different epochs. No major distortions in the wavelength are found, namely not higher than about $30 \mathrm{~m} \mathrm{~s}^{-1}$, where this limit is set by the photon noise of our observations. We do indeed reveal zero offsets in the range 0 up to $\approx 50 \mathrm{~m} \mathrm{~s}^{-1}$. With reference to similar observations performed with HARPS, we suggest that this is likely due to a nonuniform slit illumination. Attempts to use the twilight spectra to quantify the drifts induced by nonuniform illumination shows instead that twilight spectrum contains additional turbulence and motions, and therefore cannot be used as a reliable zero reference point.

The recorded spectrum does not show evidence of stretching of the wavelength scale or other instrumental effects in excess of the uncertainties induced by the wavelength calibration accuracy. In particular, the two UVES arms which are fed by two independent slits do not show signature for radial velocity offsets within the present accuracy of $30 \mathrm{~m} \mathrm{~s}^{-1}$.

This result has important implications on the search for $\Delta \alpha / \alpha$ currently performed with UVES which relies on relative shifts of absorbing lines falling on rather distant spectral regions and sometimes belonging to different arms of the spectrograph. For instance, Levshakov et al. (2007) measure a radial velocity difference of $-180 \pm 85 \mathrm{~m} \mathrm{~s}^{-1}$ between Fe II transitions falling in the two different arms of UVES, providing evidence for a variation in the fine structure constant $\Delta \alpha / \alpha=5.66 \pm 2.67 \mathrm{ppm}$. The present analysis shows that the line shift is unlikely to be produced by a misalignment of the two slits at the entrance of the two UVES arms.
The proposed technique has a general validity and can be applied to any spectrograph to perform a real-time quality control of the spectrograph performance during night time while the observations are carried on.

Acknowledgements. The asteroids observations were obtained in service mode in UVES calibration time. We are grateful to Cedric Ledoux and to all UVES operation astronomers for the careful job that has made these measurements possible. We thank also Fiorella Castelli and Cristophe Lovis for many useful discussions. Part of this work was supported by PRIN-INAF 2006. S.A.L. gratefully acknowledges the hospitality of ESO (Garching) and Osservatorio Astronomico di Trieste. This research has been supported by the RFBR grant No. 06-02-16489, by the Federal Agency for Science and Innovations grant NSh 9879.2006.2, and by the DFG project RE 353/48-1.

\section{References}

Allende Prieto, C., \& Garcia Lopez, R. J. 1998a, A\&AS, 129, 41

Allende Prieto, C., \& Garcia Lopez, R. J. 1998b, A\&AS, 131, 431

Avelino, P. P., Martins, C. J. A. P., Nunes, N. J., \& Olive, K. A. 2006, Phys. Rev. D, 74, 083508

Brault, J., \& Neckel, H. 1987, Spectral atlas of solar absolute disk-averaged and disk-center Intensity from 3290 to 12510 , unpublished. Tape-copy from KIS IDL library

Butler, R. P., Bedding, T. R., Kjeldsen, H., et al. 2004, ApJ, 600, 75

Copeland, E. J., Sami, M., \& Tsujikawa, S. 2006, Int. J. Mod. Phys., D15, 1753

Chand, H., Srianand, R., Petitjean, P., \& Aracil, B. 2004, A\&A, 417, 853

Chand, H., Srianand, R., Petitjean, P., et al. 2006, A\&A, 451, 45

de Cuyper, J.-P., \& Hensberge, H. 1988, A\&AS, 129, 409

Deming, D., \& Plymate, C. 1994, ApJ, 426, 382

Dzuba, V. A., Flambaum, V. V., Kozlov, M. G., \& Marchenko, M. V. 2002, Phys. Rev. A, 66, 022501

Fujii, Y. 2008, Phys. Lett. B, 660, 87

Gray, D. F., Tycner, C., \& Brown, K. 2000, PASP, 112, 328

Kaufer, A., D’Odorico, S., \& Kaper, L. 2004, UV-Visual Echelle Spectrograph. User Manual, http://www . eso.org/instruments/uves/userman/, 40 Kurucz, R. L., Furenlid, I. J., \& Testerman, L. 1984, NOAO Atlas No. 1, The Solar Flux Atlas from 296 to 1300 nm, Sunspot, (NM: National Solar Observatory)

Levshakov, S. A., Centurión, M., Molaro, P., \& D’Odorico, S. 2005, A\&A, 434, 827

Levshakov, S. A., Centurión, M., Molaro, P., et al. 2006, A\&A, 449, 879

Levshakov, S. A., Molaro, P., Lopez, S., et al. 2007, A\&A, 466, 1077

Lovis, C., \& Pepe F. 2007, A\&A, 468, 1115

Martins, C. J. A. P. 2006 [arXiv:astro-ph/0610665]

McMillan, R. S., Moore, T. L., Perry, M. L., \& Smith, P. H. 1993, ApJ, 403, 801

Murphy, M. T., Flambaum, V. V., Webb, J. K., et al. 2004, in Astrophysics, Clocks and Fundamental Constants, ed. S. G. Karshenboim, \& E. Peik (Berlin, Heidelberg: Springer-Verlag), 131

Murphy, M. T., Tzanavaris, P., Webb, J. K., \& Lovis, C. 2007, MNRAS, 378, 221

Murphy, M. T., Webb, J. K., \& Flambaum, V. V. 2008, MNRAS, 384, 1053

Pepe, F., Mayor, M., Queloz, D., et al. 2005, The Messenger, 120, 22

Porsev, S. G., Koshelev, K. V., Tupitsyn, I. I., et al. 2007, Phys. Rev. A, 76, 52507

Quast, R., Reimers, D., \& Levshakov, S. A. 2004, A\&A, 415, L7

Srianand, S., Chand, H., Petitjean, P., \& Aracil, B. 2007 [arXiv: 0711.1742]

Udry, S., Mayor, M., \& Queloz, D. 1999, in Precise Radial Velocities, ed. J. B. Hearnshaw, \& C. D. Scarfe, ASP Conf. Ser., 185, 367

Webb, J. K., Flambaum, V. V., Churchill, C. W., Drinkwater, M. J., \& Barrow, J. D. 1999, Phys. Rev. Lett., 82, 884

Zwitter, T., Mignard, F., \& Crifo, F. 2007, A\&A, 462, 795 